\title{
Research on the Present Situation and Strategies of Knowledge Sharing Behavior among University Teachers in Jilin Province
}

\author{
Yan Bao \\ Scientific Research Office, Jilin Engineering Normal University, Changchun, China \\ 369275690@qq.com
}

Keywords: University teachers; Knowledge sharing; Organizational structure; Incentive mechanism

\begin{abstract}
This paper analyzes the teachers' knowledge sharing willingness, sharing way, sharing satisfaction degree, sharing motivation and sharing mechanism through the way of questionnaires and in-depth interviews of 256 teachers in 6 colleges and universities in Jilin Province. Regarding to the main problems existing in the university teachers' knowledge sharing from the aspects of organizational structure, campus culture, incentive mechanism, technology platform and self - ability, this article will be present an effective strategy for promoting knowledge sharing among university teachers.
\end{abstract}

\section{Introduction}

The development of advanced education needs the support of high-qualified talent team, especially for the innovative faculty. The innovation ability of teachers is not only from their own strong knowledge reserves, but also need high frequency of knowledge sharing. Through the way of sharing knowledge can continuously enrich their knowledge base, thereby enhancing the teaching and research capabilities for university teachers. For example, a research project, it is generally difficult to be completed by the individual teachers alone, but the need for the same discipline, similar disciplines and even different disciplines members of the mutual cooperation and collaboration, this is also the objective rules of scientific research requirements. In the reality of teaching and research practice, many colleges and universities exists the situation of knowledge sharing limitations generally. Most of the university teachers are reluctant to share the most important knowledge assets with others, so that a large amount of knowledge cannot be transferred and effectively used among teachers or between teachers and students.

\section{Investigation on the Current Situation of University Teachers' Knowledge Sharing Behavior}

In order to deeply and objectively understand the present status and obstacles of university teachers' knowledge sharing, we are selecting 6 colleges and universities in Jilin Province, including 1 deployment university and 5 provincial universities. With the combination way of interview survey and questionnaire survey, deeply understand of the status of knowledge sharing motivation, knowledge sharing method, knowledge sharing satisfaction and knowledge sharing mechanism, and so on. The questionnaire used the Likert 5-point scale model, from 1 to 5 level, respectively represent from the "very disagree" to "very agree" five identity level. In order to ensure the objectivity and scientific nature of the survey results, we initiated a small-scale of pre-survey before the formal investigation, and revised the questionnaire according to the conclusions of the pre-survey. For the formal investigation of 6 colleges and universities, a total of 315 survey questionnaires were issued and received of 256 effective questionnaires. Among all the subjects under investigation, including 90 male teachers and 166 female teachers, the structure of the ratio is shown by the Fig. 1; including 113 teachers of liberal arts and 143 science teachers, the structure is shown by the Fig. 2; including 42 high level title teachers, 58 vice title teachers, 123 lecturers and others of 33 teachers, the structure of the ratio is shown by the Fig. 3; The age and gender two-dimensional effective sample number distribution is shown by Table 1 . 

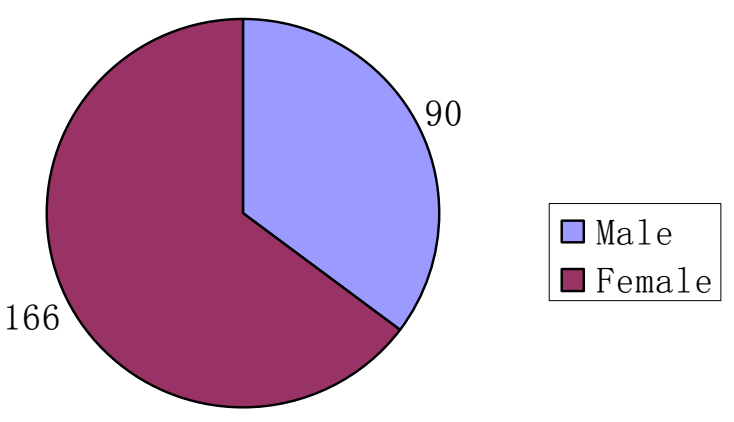

Figure 1. Distribution of the number of valid samples of gender

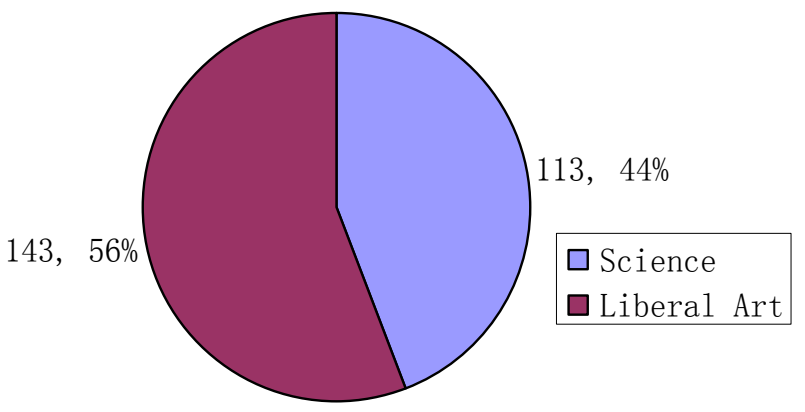

Figure 2. Distribution of the effective sample size of the surveyed objects

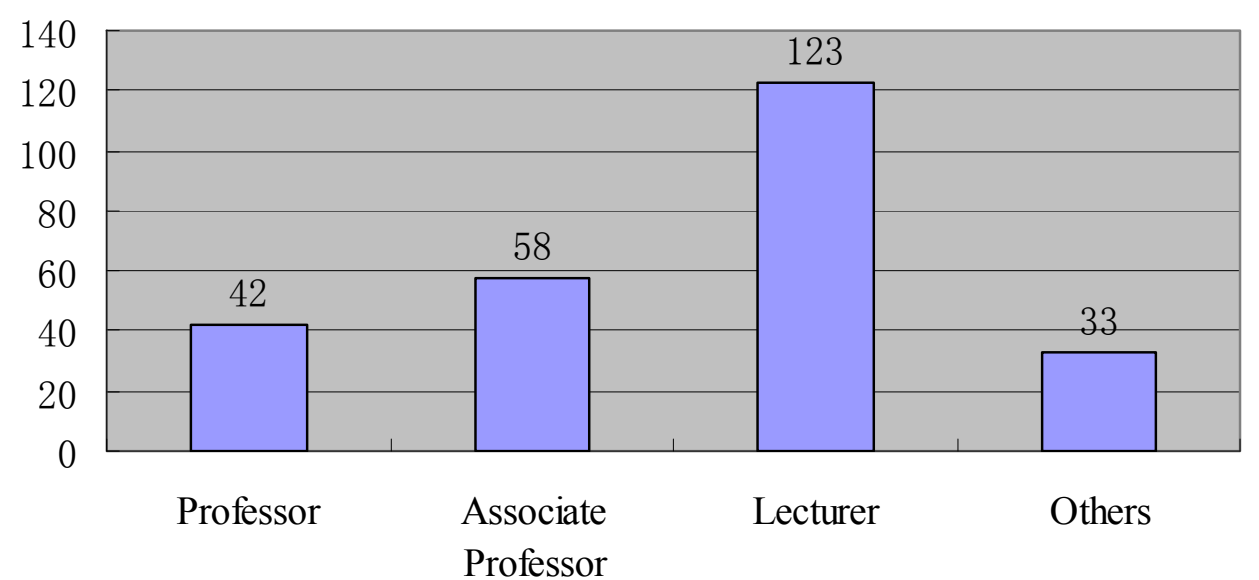

Figure 3. Distribution of the number of valid samples for the title of surveyed subjects

Table 1 Distribution two-dimensional effective sample of Age and Sex

\begin{tabular}{|c|c|c|c|}
\hline \multirow{2}{*}{ Age (year) } & \multicolumn{2}{|c|}{ Sex } & \multirow{2}{*}{ Total } \\
\cline { 2 - 3 } & Male & Femal & 60 \\
\hline $20-30$ & 28 & 32 & 99 \\
\hline $31-40$ & 35 & 64 & 76 \\
\hline $41-50$ & 20 & 56 & 16 \\
\hline $51-60$ & 6 & 10 & 5 \\
\hline More than 61 & 1 & 4 & 256 \\
\hline Total & 90 & 166 & \\
\hline
\end{tabular}




\section{The Analysis on University Teachers' Knowledge Sharing Status}

Based on the results of questionnaire survey on the knowledge sharing willingness, knowledge sharing method, knowledge sharing satisfaction, knowledge sharing motivation and knowledge sharing platform, we found there are significant differences for teachers in colleges and universities regarding to the sharing knowledge causing by the reason of work experience and professional ranks and titles.

Not High of Knowledge Sharing Intention. By this survey, we found that most college and university teachers are very keen to gain knowledge through the sharing of knowledge, more than $50 \%$ of the respondents believe that knowledge sharing for college and university teachers are "very important", but less than $10 \%$ of respondents are willing to share knowledge to others. At the same time, we also found that the amount of teachers who are willing to share knowledge are mostly conditional to share with others, such as based on "do not touch their own interests", "through exchange knowledge can get what they want other knowledge" and so on. Thus, college and university teachers' sharing knowledge willingness is not very high behind of their strong request for knowledge sharing in the meantime.

A Single Way of Knowledge Sharing. According to the survey, we found that the sharing of knowledge is relatively simple among of the university teachers. Language communication and information exchange are the most commonly used methods of knowledge sharing under this investigation, $68.5 \%$ of the survey respondents believe that personal contact, especially the language of communication and information exchange can better conduct explicit and tacit knowledge sharing, especially for tacit knowledge sharing, because the full communication and information exchange between individuals is more conducive way to understand of knowledge to achieve a doubling of the amount of individual knowledge.

The Knowledge Sharing Satisfaction is Low. Through the result of field interviews and questionnaires, we found that teachers' knowledge sharing satisfaction is generally low. Of the total amount of 256 teachers surveyed, only $4.6 \%$ were "very satisfied" with knowledge sharing; only $9.8 \%$ felt "satisfied"; $17.3 \%$ felt "generally satisfied"; Respectively, $39.4 \%$ and $28.9 \%$ of the teachers were "less satisfied" and "very dissatisfied" with knowledge sharing as shown by the Fig. 4.

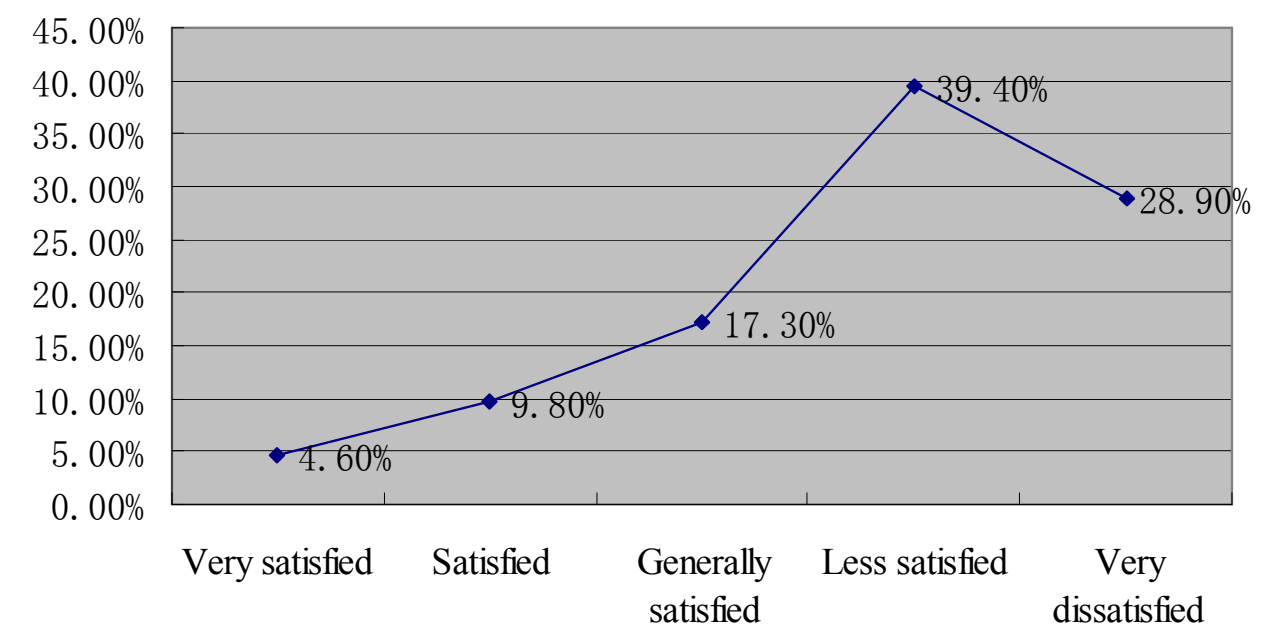

Figure 4. Distribution of the number of valid samples for the title of surveyed subjects

Clear Motivation of Knowledge Sharing. According to Maslow's "five-level" needs theory, we require the respondents to make the order of importance in the motivation of knowledge sharing. Data analysis showed that $78.4 \%$ of teachers chose "self-actualization needs". Thus, the pursuit of personal growth and career success and other spiritual satisfaction is an important motivation for university teachers to share knowledge, and almost no respondents selected "physiological needs", which is in line with the nature of the work of teachers, their characteristics and corresponding of the values. 
Moreover, with the increasing of the work experience of the respondents, they are "self-actualization needs" knowledge sharing motivation is more and more recognition.

Lack of Knowledge Sharing Mechanism. A strengthen and efficient knowledge sharing platform is conducive to improving the speed of knowledge sharing and quality between teachers. But in the survey, we found that many university teachers believe that the university's knowledge-sharing platform construction existing some certain limitations. $48.6 \%$ of teachers believe that the university does not have a perfect knowledge-sharing incentive mechanism, such as requiring of associate professors or more titles of teachers held at least once a year academic lectures, but the university does not give any reward. If teachers are fail to complete, will be deducted as part of the allowance eventually. $67.4 \%$ of the teachers thought that the university did not establish the effective knowledge media and the channel, for example, the teacher cannot go to the "China Knowledge Network" at home, and teachers can only download the academic data in the campus. It has greatly affected the teacher's academic research interest and the research result. Nearly $50 \%$ of the teachers indicated that the construction of the knowledge-sharing environment and the campus culture was not strong enough. Some academic activities were only focusing on the form and teachers did not get much benefit for the knowledge sharing.

\section{Summary}

In view of the main problems in the knowledge sharing of university teachers, it is necessary to put forward effective strategies to promote the knowledge sharing among university teachers from the aspects of organizational structure, campus culture, incentive mechanism, technology platform and self - ability.

To Construct a Flexible Organizational Structure. In one hand, the flexibility of the organizational structure allows colleges and universities to flexibly respond the changes of external environment. As shown by the Fig. 5, under the president unified instruction, the faculty and subject team of professional teachers to conduct academic communication and research, the research achievement is owned by the Department and the academic team; it can greatly enhance the knowledge sharing effect. On the other hand, a flexible organizational structure allows teachers from different faculties and discipline-team to work together to increase the tolerance and understanding. Interdisciplinary research is not only easy to solve scientific problems, and also easy way to get creative achievement, so that it will be promote the faculty and research team cooperation more smoothly for sharing of knowledge and further spread to lay a good organizational environment.

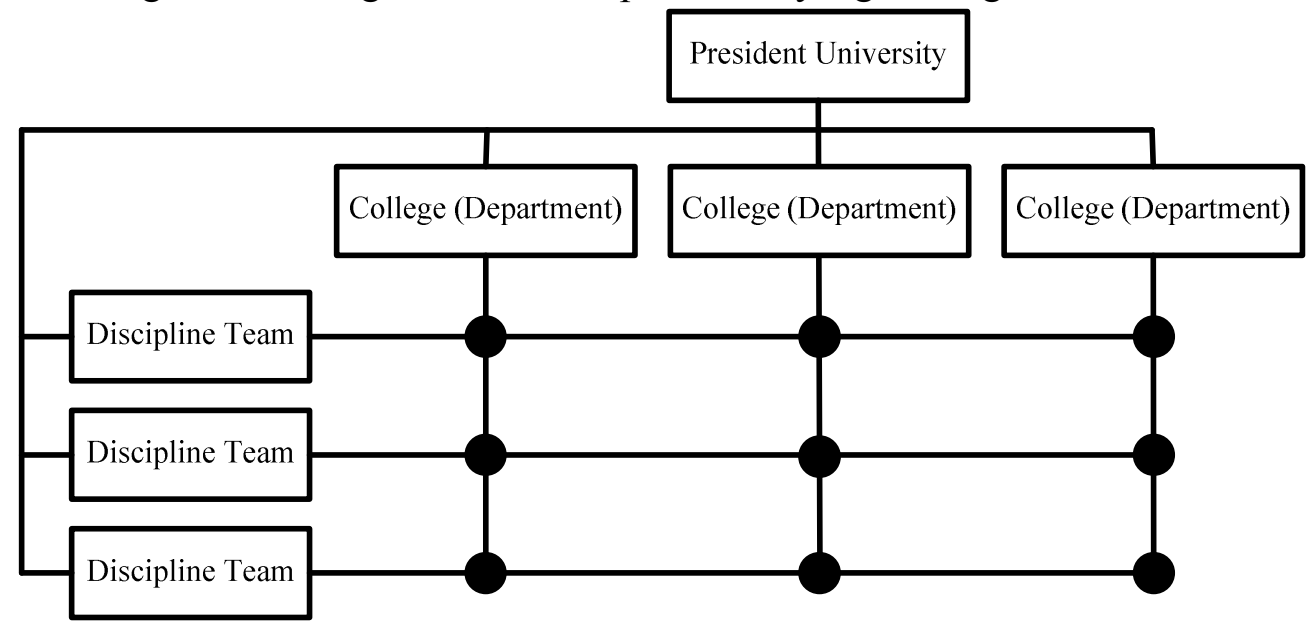

Figure 5. Flexible organization structure

To Create a Harmonious Campus Culture. Harmony and integrity of the campus culture atmosphere is a prerequisite to protect the smooth sharing of knowledge of teachers in colleges and universities, it can provide a common knowledge sharing of teachers in colleges and universities, 
values and norms. In a culture of mutual trust, teachers do not have to worry about their own intellectual property infringement by others or seize another person's belongings; you can communicate knowledge equally with others without worry about teaching experience and academic research experience. Knowledge will be present in the form of text on the basis of mutual trust and equality to achieve efficient interaction of knowledge to increase each other's benefits and effectiveness of knowledge sharing.

To Improve the Knowledge Sharing Incentive Mechanism. Knowledge sharing intention is driven by knowledge sharing motivation. A strengthen and efficient mechanism is an effective driving force to promote knowledge sharing among university teachers. Colleges and universities should establish a reasonable incentive mechanism for knowledge sharing from the internal drive and external drive two aspects of human nature design, such as providing free network resources for teachers, and then they can always get the required research information, search various of teaching resources; providing a higher self-efficacy of teachers to enhance the professional development of space and platform; providing an outstanding teachers provide a variety of material incentives and spiritual incentives in the workplace. Through the establishment of perfect knowledge incentive mechanism, and constantly make outstanding talent come to the fore, so that teachers can feel the value of knowledge sharing, and sharing their achievement to others selflessly.

To Construct the Knowledge Sharing Technology Platform. Knowledge sharing platform is to speed up the knowledge sharing of the carrier of university teachers, but also indispensable technical method and guarantee. Knowledge-sharing platform can be constructed by constructing knowledge map, knowledge base and teacher organization intranet. Such as the construction of campus network resources, free open all kinds of databases; through e-learning learning platform, so that each teacher's teaching resources can use and reference for other teachers and students, to minimize the knowledge sharing in time and space distance; through the knowledge map is given the school various newspapers, libraries, documents, software libraries, archives and other explicit knowledge of the storage location, it is much easier to search for teachers. By building a knowledge-sharing platform, teachers can greatly enhance the efficiency of knowledge-sharing and effectiveness.

To Enhance the Ability of Knowledge Sharing among Teachers. The university has the multi-function of personnel training, scientific research and social service. This requires teachers' work itself to be a process of continual knowledge-sharing. Therefore, teachers should be as the main body of knowledge sharing, should continue to learn in practice, and actively enhance their knowledge reserves and knowledge-sharing capabilities. In the future, teachers can apply for a higher degree or apply for a visiting scholar and abroad to do to visit the enterprise to learn and develop a variety of practical skills, to consult with relevant stakeholders, to constantly try to figure out and practice, make the tacit knowledge be explicit, and gradually into the teaching work; the formation of individual sites, and more to communicate with their peers, to participate in various academic exchanges will be sharing their own teaching experience, sentiment and reflection with others in order to continuously enhance their knowledge-sharing capabilities.

\section{Acknowledgements}

This work was financially supported by Social Science Foundation of Jilin Provincial Education Department ([2014] No.415) and Scientific Research Office of Jilin Engineering Normal University.

\section{References}

[1] J. Chennamaneni: Determinants of Knowledge Sharing Behaviors: Developing and Testing an Integrated Theoretical Model (Ph.D., The University of Texas at Arlington, USA 2006), p.13.

[2] P. Hendriks: Knowledge and Process Management, Vol. 6 (1999) No.2, p.91-100.

[3] I. Nonaka, H. Takeuchi: The Knowledge Creating Company (Trans Oxford University Press, USA 1995). 
[4] S. Taylor, P. Todd: International Journal of Research in Marketing, Vol. 12 (1995) No.2, p.137-155.

[5] W.B. Lin: Expert Systems with Applications, Vol. 35 (2008) No.1, p.661-676.

[6] R. Xu: The Research on the Relationship among Organizational Climate and Internal Social Capital and Knowledge-sharing Behavior (Ph.D., Southwestern University of Finance and Economics, China 2012), p.20-21.

[7] S.P. Chen: Journal of Guangzhou University (Social Science Edition), Vol.26 (2012) No.7, p.69-72.

[8] Q.L. Luo, X.Y. Pang and C.Y. Wang: Information Science, Vol. 33 (2013) No.7, p.55-58.

[9] H.Y. Luo, X.Y. Lin and Y.F. Gong: Information Science, Vol. 30 (2010) No.3, p.116-119.

[10]J.Qiang and Q. Mei: Science and Technology Management Research, Vol. 30 (2010) No.4, p.248-251.

[11] Y.C. Tao and Y.M. Xie: Science \& Technology Progress and Policy, Vol. 25 (2008) No.12, p.5-8.

[12] L. Wang and D.H. Wu: Research on Library Science, Vol. 29 (2010) No.2, p.94-97. 